\title{
Near field plasmon and force microscopy
}

\author{
R.B.G. de Hollander N.F. van Hulst ${ }^{*}$, R.P.H. Kooyman \\ Applied Optics Group, Department of Applied Physics, University of Twente, P.O. Box 217, 7500 AE Enschede, The Netherlands
}

Received 10 November 1993; accepted 7 January 1994

\begin{abstract}
A scanning plasmon near field optical microscope (SPNM) is presented which combines a conventional far field surface plasmon microscope with a stand-alone atomic force microscope (AFM). Near field plasmon and force images are recorded simultaneously both with a lateral resolution limited by the probe size to about $20 \mathrm{~nm}$. At variance to previous work, utilizing a scanning tunneling microscope (STM) with a metallic tip, a dielectric silicon-nitride tip is used in contact mode. This arrangement is more suitable for biolngical and chemical samples. Approaching the tip towards the surface the exponential behaviour of the evanescent field and interference between scattering at the tip and the surface are observed. SPNM and AFM images of gold grains show strong correlation induced by the topography. Images of a transition between two different dielectric areas show optical contrast in the SPNM image which cannot be obtained from the topographic force image. Contrast in the SPNM images can be adjusted, or even inverted, by choosing proper surface plasmon excitation conditions. Characteristic fringe patterns are observed caused by interference between the evanescent wave propagating along the surface and scattering of the surface plasmon at larger objects.
\end{abstract}

\section{Introduction}

Optically excited surface plasmons (SP) are widely being applied to enhance the sensitivity in studies of metal surfaces and dielectric thin films. The resonance conditions which cause strong localization and field enhancement of the SP at the metal surface are strongly dependent on surface roughness, refractive index and thickness of any adsorbate layer. The sensitivity can be sub-monolayer in thickness and $10^{-4}$ in refractive index. This high sensitivity is exploited in surface plasmon microscopy (SPM) as demonstrated by sev-

\footnotetext{
* Corresponding author.
}

eral groups [1-3]. SPM is especially an attractive technique for the study of monolayers, as it allows observation of phase segregation based on the different dielectric properties of different domains. In contrast to its thickness resolution, the lateral resolution of SPM is fairly limited due to the finite SP propagation length, in the order of microns, and the limited accessibility of the surface under study for high NA objectives. A near field version of the SPM with a localized probing of the field enhancement conditions could improve the lateral resolution to the nanometer regime. Recently Marti et al. [4], Safarov et al. [5] and Dawson et al. [6] have directly probed the SP induced field enhancement in a photon scanning tunneling microscope (PSTM) configuration, where indeed up to two orders of magnitude field 
enhancement is observed in accordance with theoretical expectations. First SPNM images were obtained by Specht et al. [7] by measuring the change in the attenuation of the light intensity reflected from a metal surface (ATR configuration) caused by a metal tip tunneling to the surface. Simultaneously STM and SPNM were obtained with $3 \mathrm{~nm}$ lateral resolution. In order not to limit ourselves to conducting surfaces we have chosen to combine a SPM with an atomic force microscope (AFM) using a dielectric silicon-nitride $(\mathrm{SiN})$ probe which is more suitable for chemical and biological applications. The SP resonance is frustrated by the SiN tip similar to the situation in our PSTM configuration using a SiN probe as reported earlier [8]. In principle both detection in reflection and transmission are possible. In this paper operation of SPNM in reflection is presented.

\section{Experimental set-up}

The microscope is schematically shown in Fig. 1. The experimental set-up is based on a conventional Kretschmann-Raether configuration with a Au film on a $\mathrm{K} 5$ prism where a SP is excited by a parallel $\mathrm{HeNe}$ laser beam, narrowed to $300 \mu \mathrm{m}$, incident at the matching angle with p-polarization. The set-up contains four microscopes. (i) An SPM: the reflected beam is monitored by a CCD camera using a $4 \times, 0.12$ NA objective and a telescope system. Angles of incidence and reflectance are adjustable. Imaging on the CCD camera constitutes essentially a conventional SPM with high sensitivity for variations in dielectric constant and thickness and a lateral resolution of about $5 \mu \mathrm{m}$. (ii) An AFM: on top of the prism is a stand-alone atomic force microscope [9], using a microfabricated SiN cantilever probe with about $20 \mathrm{~nm}$ tip apex. The tip is scanned over a maximum scan area of $30 \times 30 \mu \mathrm{m}^{2}$ using a piezo-tube. The tip-sample interaction force is detected by an optical beam deflection system, where both cantilever bending and torsion are monitored. Thus both vertical and lateral force images are obtained. (iii) The SPNM: the $20 \mathrm{~nm} \mathrm{SiN} \mathrm{apex}$ changes locally the conditions for SP resonance. The effect on the local reflectance of the scanning SiN probe is monitored on a PMT. A pinhole is used to limit the area over which the reflectance signal is detected by the PMT,

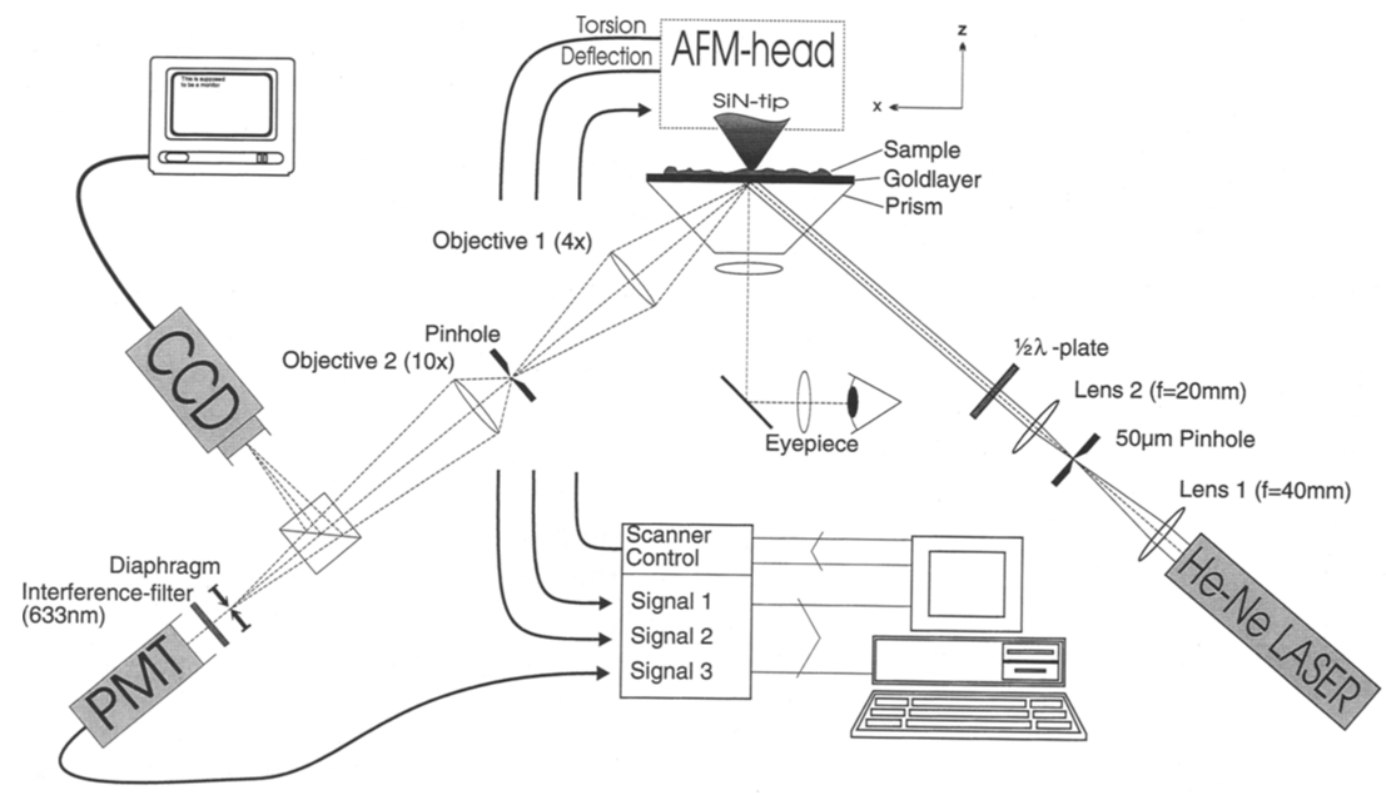

Fig. 1. Experimental arrangement of the scanning plasmon near field optical microscope (SPNM). 
whereas the pinhole is large enough to cover the scan range of the probe. (iv) An alignment microscope: an extra conventional microscope is installed with its optical axis perpendicular to the sample surface, which facilitates alignment of the optical beam deflection system, the plasmon excitation area and selection of a specific sample area. After choice of proper angles of incidence and reflectance and alignment of the pinhole relative to the tip the probe is scanned and SPNM and AFM images are obtained simultaneously.

\section{Results}

First approach curves have been recorded to obtain some insight in the tip-sample interaction, see Fig. 2. The sample is a $46 \mathrm{~nm}$ Au layer $(\varepsilon=-11.7+\mathrm{i} 1.4)$ evaporated on a $\mathrm{K} 5$ cover glass
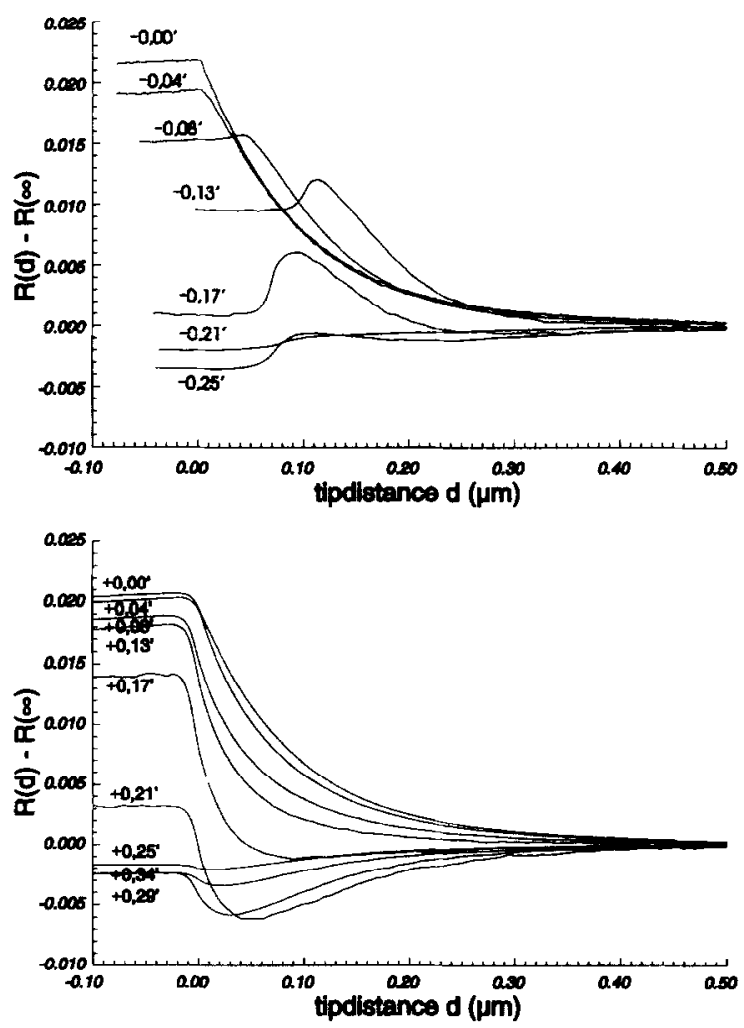

Fig. 2. Reflectance change $R(d)-R(\infty)$ as a function of tip-sample distance $d$ for several angles of incidence $\vartheta_{i}(\mathrm{deg})$ around $\vartheta_{\mathrm{sp}}$, where $\Delta \vartheta=\vartheta_{\mathrm{i}}-\boldsymbol{\vartheta}_{\mathrm{sp}}$. $(\varepsilon=2.31)$. For this system the angle of SP excitation $\left(\vartheta_{\mathrm{sp}}\right)$ is at $\vartheta_{\mathrm{sp}}=43.4^{\circ}$ with $0.3^{\circ}$ plasmon dip width and a minimum reflectance value of $R_{\text {min }}$ $=0.013$ at resonance. Fig. 2 displays the reflectance change $(\Delta R=R(d)-R(\infty))$ as a function of tip-sample distance $d$ for several angles of incidence $\vartheta_{\mathrm{i}}$ around $\vartheta_{\mathrm{sp}}$. Maximum SP disturbance is observed at $\Delta \vartheta=\vartheta_{\mathrm{i}}-\vartheta_{\mathrm{sp}}=0.0^{\circ}$, with an exponential behaviour for increasing $d$, corresponding to the decay of the evanescent wave. For positive $\Delta \vartheta$ values a minimum in the reflectance is found at $d=50 \mathrm{~nm}$ and for negative $\Delta \vartheta$ a maximum is observed around $d=100 \mathrm{~nm}$. At $d=0$ the tip is in contact with the surface and further approach only bends the cantilever but does not affect the observed reflectance. On retracting the probe from the surface the tip tends to stick to the surface due to adhesion caused by a contamination layer, therefore retraction curves display an abrupt jump to out of contact. Preliminary calculations, using a stratified layer model, show correspondence with the observed trends in the distance dependence, however tip geometry does play an important role and requires further improvement of the model.

Fig. 3 displays simultaneously obtained AFM and SPNM images of a $600 \times 600 \mathrm{~nm}^{2}$ area of the $46 \mathrm{~nm} \mathrm{Au}$ layer at $\Delta \vartheta=0.0^{\circ}$ and a $1.2 \times 1.2 \mu \mathrm{m}^{2}$ area at $\Delta \vartheta \approx-0.10^{\circ}$. The force images in height and torsion mode display the gold corrugation with grains typically $50 \mathrm{~nm}$ in size. AFM resolution is about $20 \mathrm{~nm}$ lateral and $0.5 \mathrm{~nm}$ vertical. The SPNM image at $\Delta \vartheta=0.0^{\circ}$ (Fig. 3c) displays a variation in reflectance of 0.0045 over an average reflectance of 0.045 , i.e. $10 \%$ contrast, and correlates strongly with the height image (Fig. 3a). The reflected light level decreases with increasing distance between the tip and average sample plane, consequently grains appear dark. SPNM lateral resolution is also $\sim 20 \mathrm{~nm}$, and seems to be determined by the tip size. The SPNM image at $\Delta \vartheta \approx-0.10^{\circ}$ (Fig. 3f) shows contrast reversal, where again the Au grains correlate to the corresponding regions in the AFM image (Fig. 3d). Contrast is rather low, less than $1 \%$, as the reflectance varies only over 0.002 on an average value of 0.23 . The contact-mode AFM images are conventionally interpreted as due to 

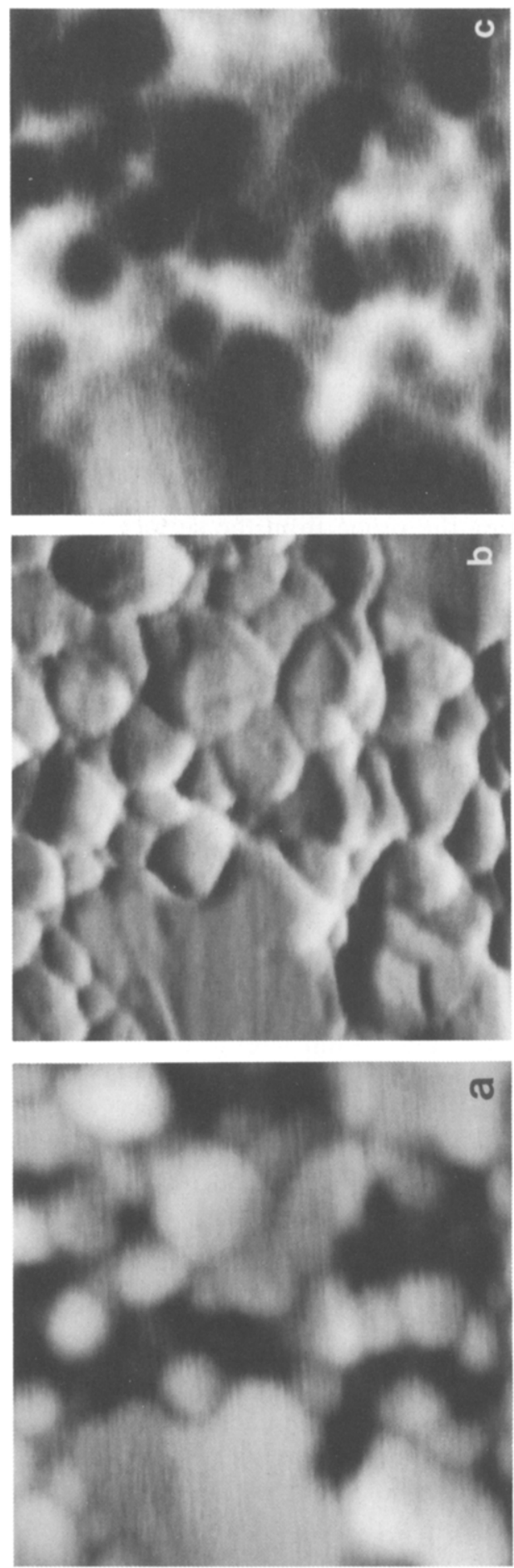
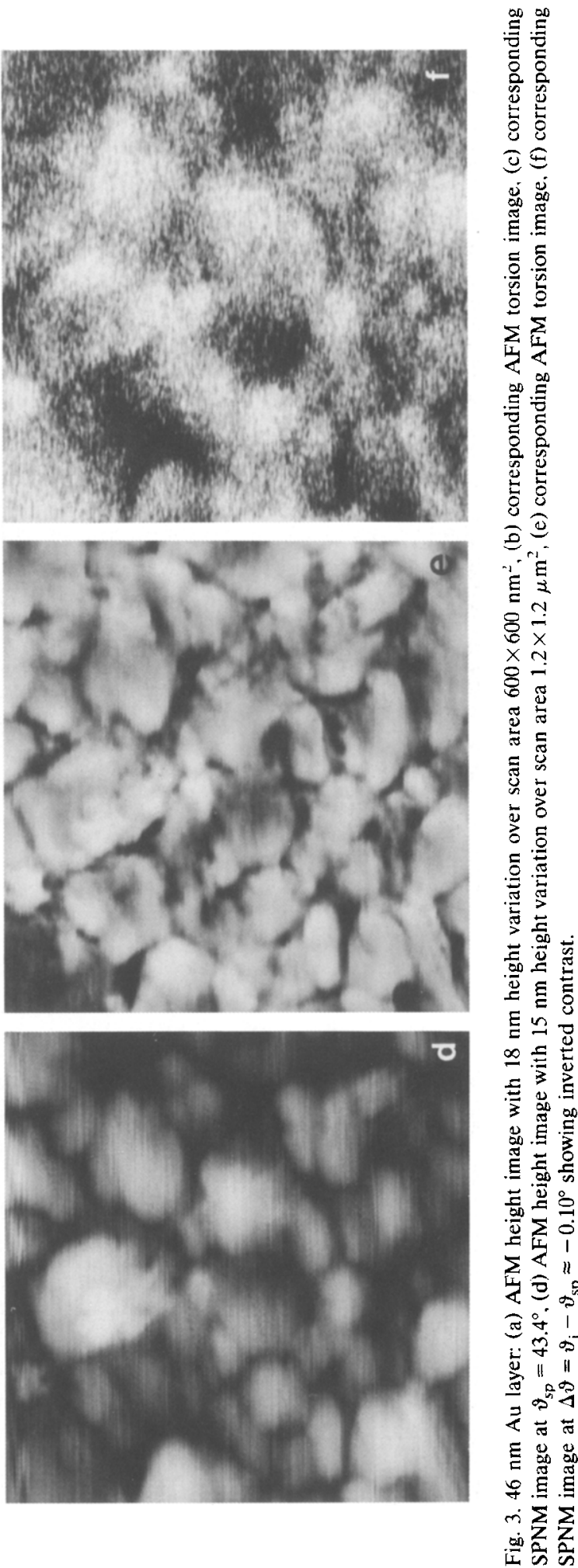

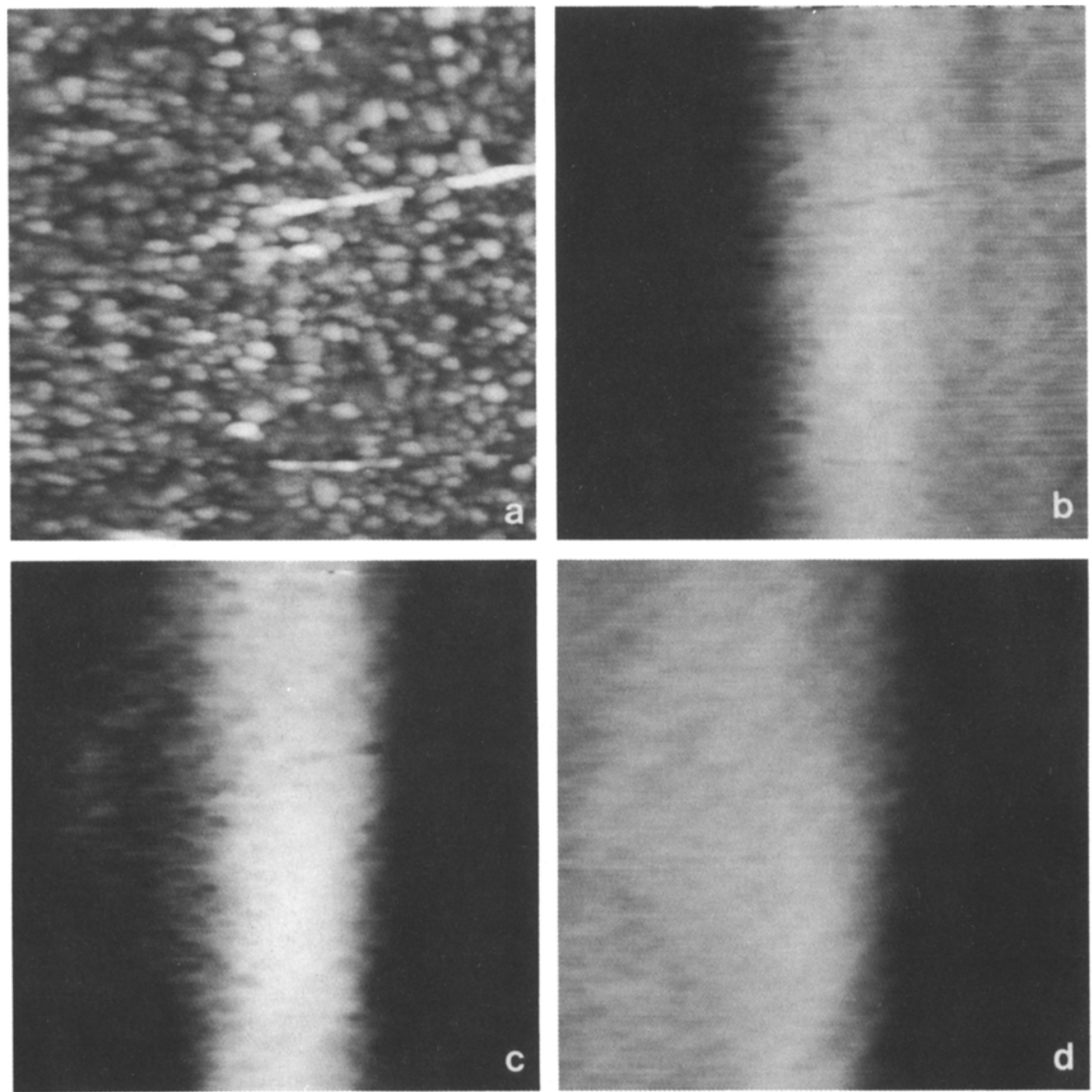

Fig. 4. Edge between $46 \mathrm{~nm}$ Au layer (left) and $4 \mathrm{~nm} \mathrm{SiO}{ }_{2}$ overcoat. Image size $5 \times 5 \mu \mathrm{m}^{2}$. Surface plasmon excitation direction vertical. AFM height image (a) and corresponding SPNM images at angle of incidence $\vartheta_{i}=43.8^{\circ}$ (b), $43.7^{\circ}$ (c) and $43.6^{\circ}$ (d).
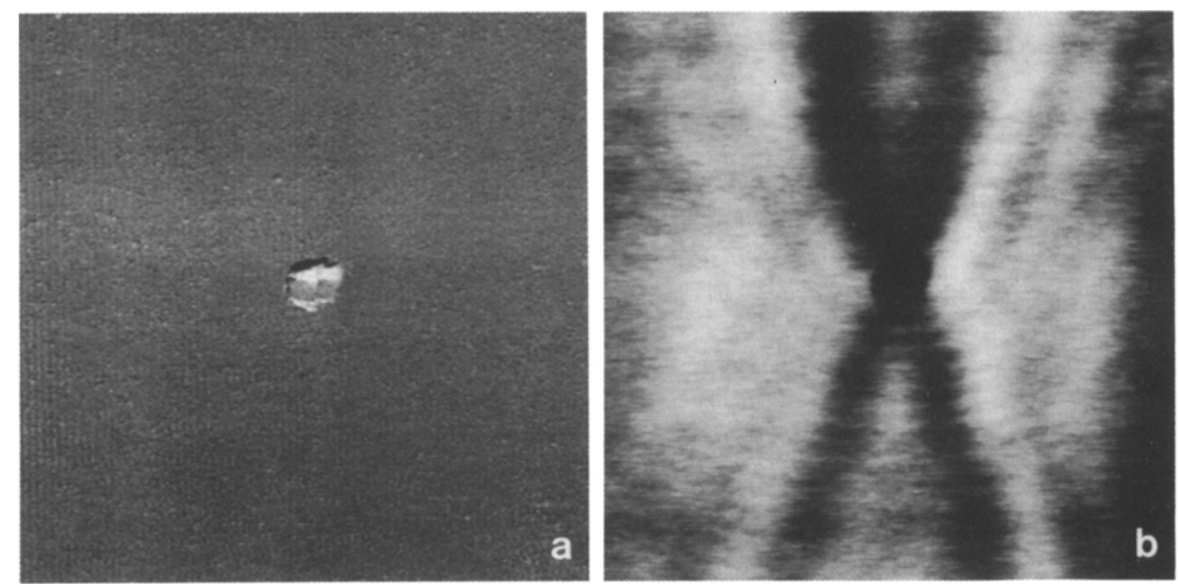

Fig. 5. Single particle on $46 \mathrm{~nm}$ Au layer. Scan area $6 \times 6 \mu \mathrm{m}^{2}$. AFM torsion (a) and corresponding SPNM image (b) at $\vartheta_{\mathrm{sp}}=43.6^{\circ}$. 
topographic contrast, therefore, in view of the high correlation between AFM and SPNM images, the high-resolution SPNM details are caused mainly by topographic and not dielectric effects.

Fig. 4 shows AFM and SPNM images $(5 \times 5$ $\mu \mathrm{m}^{2}$ ) of an edge of a $4 \mathrm{~nm} \mathrm{SiO}_{2}$ cover layer, evaporated on $46 \mathrm{~nm} \mathrm{Au}$. Covered and uncovered areas have a different SP excitation angle of $43.8^{\circ}$ and $43.6^{\circ}$, respectively. In the images bare gold is at the left side and the $\mathrm{SiO}$, layer at the right side. The $4 \mathrm{~nm}$ step is hardly visible in the AFM image (Fig. 4a) and merely shows the Au corrugation. SPNM images, Fig. $4 b, 4 c$ and $4 d$, are taken at $\vartheta_{\mathrm{i}}=43.8^{\circ}, 43.7^{\circ}$ and $43.6^{\circ}$, respectively. The transition between $\mathrm{Au}$ and $\mathrm{SiO}_{2}$ is clearly visible, where the area excited at SP resonance shows up light. From SEM data (not shown) we know that the width of the transition area, about $1 \mu \mathrm{m}$, is determined by the masking and etching in the edge fabrication. The SPNM images in Fig. 4 are dominated by the difference in dielectric constant in both areas and still show some high-resolution topography.

Fig. 5 shows a $6 \times 6 \mu \mathrm{m}^{2}$ AFM and SPNM image of a $0.5 \mu \mathrm{m}$ particle (gold cluster or dust) on $46 \mathrm{~nm} \mathrm{Au}$, with the incident beam propagating in vertical direction. In the SPNM image a characteristic X-shaped pattern is observed which appears to be common for larger objects $(\geqslant \lambda)$. Upon closer inspection a periodic fringe pattern is distinguished with $300 \mathrm{~nm}$ period caused by interference between waves scattered at the par-
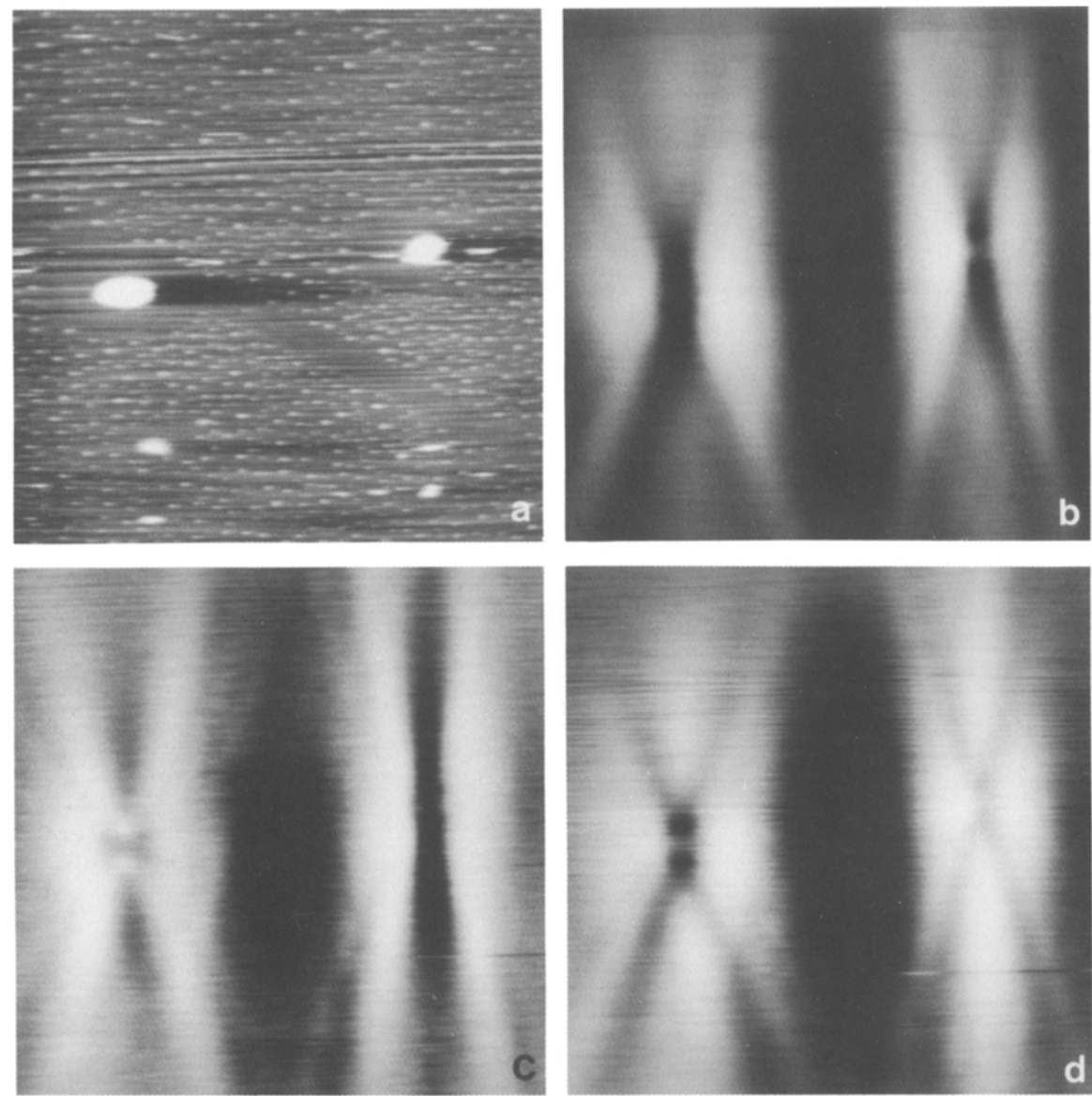

Fig. 6. Two particles on $46 \mathrm{~nm}$ Au layer. Scan area $18 \times 18 \mu \mathrm{m}^{2}$. AFM height image (a) and corresponding SPNM images at different angles of incidence, $\Delta \vartheta=0.0^{\circ}(\mathrm{b}), \Delta \vartheta=-0.42^{\circ}$ (c) and $\Delta \vartheta=0.20^{\circ}$ (d). 


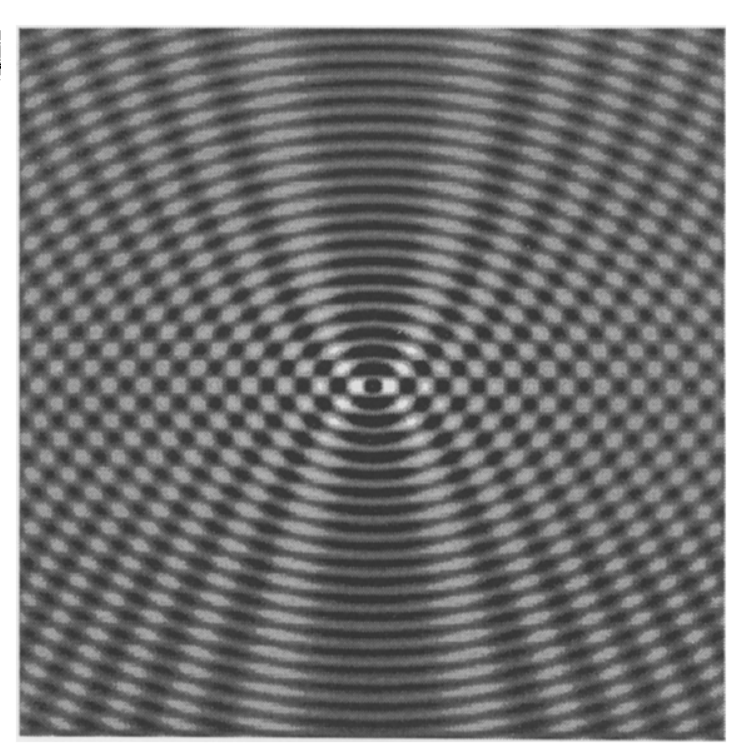

Fig. 7. Calculated interference pattern for a single point object. The area size is 20 wavelengths.

ticle and at the tip. Fig. 6 also shows AFM and SPNM images $\left(18 \times 18 \mu \mathrm{m}^{2}\right)$ of two particles, different in size on $46 \mathrm{~nm}$ Au. SPNM images in Figs. $6 \mathrm{~b}, 6 \mathrm{c}$ and $6 \mathrm{~d}$ are taken at $\Delta \vartheta=0.0^{\circ},-0.42^{\circ}$ and $0.20^{\circ}$, respectively, with the beam propagating in the vertical direction. Changing the excitation angle affects the phase between the reflected field and the field scattered at the tip, which results in a contrast inversion in the interference pattern. The interference patterns can be modeled by assuming isotropic scattering of SP waves at both tip and object along the surface. Considering phase and amplitude of the scattered waves for each position of the tip around the object and calculating the superposition of the plasmon field and the two scattered waves the expected fringe pattern can be simulated, as shown in Fig. 7. The calculated image shows great resemblance to the observed X-shape and periodic patterns. Difference can be due to anisotropic scattering, damping of waves, tip geometry, etc. which are not included in the simple model.

\section{Conclusions}

The combined SPNM and AFM yields simultaneously high-resolution images of plasmon reso- nance disturbance and surface topography with a lateral resolution limited by tip size to $\sim 20 \mathrm{~nm}$. The optical contrast in the SPNM images is strongly sensitive to dielectric properties of the sample surface, but high-resolution features correlate strongly with the surface topography. Interpretation of the contrast mechanism in SPNM images is as yet not straightforward and requires further detailed theoretical study. Scanning at constant average height could be an approach to decouple the topographic from the true optical contrast and to test the applicability of the simplified stratified layer model that seems to describe the approach curves. Contrast inversion is observed at off-resonance conditions depending on the angle of illumination. For large size objects the SPNM images are dominated by far-field scattering resulting in characteristic interference phenomena. These interference effects can be a serious limitation of the technique in the study of high relief sample surfaces. Use of AFM instead of STM makes the SPNM more suitable for imaging of chemical and biological surfaces, specifically monolayers (Langmuir-Blodgett films) which will be the next subject of study.

\section{References}

[1] E. Yeatman and E.A. Ash, Electron. Lett. 23 (1987) 1091.

[2] B. Rothenhäusler and W. Knoll, Nature 332 (1988) 615.

[3] R.P.H. Kooyman and U.J. Krull, Langmuir 7 (1991) 1506; H.E. de Bruin, R.P.H. Kooyman and J. Greve, Appl. Opt. 32 (1993) 2426.

[4] O. Marti, H. Bielefeldt, B. Hecht, S. Herminghaus, P. Leiderer and J. Mlynek, Opt. Commun. 96 (1993) 225.

[5] V.I. Safarov, V.A. Kosobukin, C. Hermann, G. Lampel, C. Marlière and J. Peretti, Ultramicroscopy 57 (1995) 270.

[6] P. Dawson, K.W. Smith, F. de Fornel and J.-P. Goudonnet, Ultramicroscopy 57 (1995) 287.

P.M. Adam, L. Salomon, F. de Fornel and J.-P. Goudonnet, paper presented at this conference (NFO-2).

[7] M. Specht, J.D. Pedarnig, W.M. Heckl and T.W. Hänsch, Phys. Rev. Lett 68 (1992) 476;

J.D. Pedarnig, M. Specht, W.M. Heckl and T.W. Hänsch, Appl. Phys. A 55 (1992) 476.

[8] N.F. van Hulst, M.H.P. Moers, O.F.J. Noordman, R.G. Tack, F.B. Segerink and B. Bölger, Appl. Phys. Lett. 62 (1993) 461;

M.H.P. Moers, R.G. Tack, N.F. van Hulst and B. Bölger, J. Appl. Phys. 75 (1994) 1254.

[9] K.O. van der Werf, C.A.J. Putman, B.G. de Grooth, F.B. Segerink, E.H. Schipper, N.F. van Hulst and J. Greve, Rev. Sci. Instrum. 64 (1993) 2892. 